\title{
Discovery of rafoxanide as a dual CDK4/6 inhibitor for the treatment of skin cancer
}

\author{
XINAN SHI ${ }^{1-3}$, HONGJIAN LI ${ }^{4,5}$, ANHUA SHI $^{2}$, HONG YAO $^{6}$, KUNBIN KE $^{3,9}$, CHAO DONG $^{3,7}$, YING ZHU $^{3,8}$,

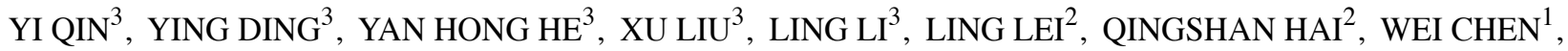 \\ KWONG-SAK LEUNG ${ }^{4,5}$, MAN-HON WONG ${ }^{5}$, HSIANG-FU KUNG ${ }^{3}$ and MARIE CHIA-MI LIN ${ }^{10}$
}

\author{
${ }^{1}$ Department of Medicine, Southwest Guizhou Vocational and Technical College for Nationalities, Xingyi, Guizhou 562400; \\ ${ }^{2}$ Department of Physiology and Pathology, Yunnan University of Traditional Chinese Medicine; \\ ${ }^{3}$ Biomedical Engineering Research Center, Kunming Medical University, Kunming, Yunnan 650504; \\ ${ }^{4}$ Institute of Future Cities; ${ }^{5}$ Department of Computer Science and Engineering, The Chinese University of Hong Kong, \\ Hong Kong 999077; ${ }^{6}$ Jiangsu Cancer Biotherapy Institute, Xuzhou Medical College, Xuzhou, Jiangsu 221000; \\ ${ }^{7}$ Department of The Second Medical Oncology, The Third Affiliated Hospital of Kunming Medical University, \\ Yunnan Tumor Hospital; ${ }^{8}$ Department of Cadre Medical Branch, The Third Affiliated Hospital of Kunming Medical University; \\ ${ }^{9}$ Department of Urology, The First Affiliated Hospital of Kunming Medical University, Kunming, Yunnan 650118; \\ ${ }^{10}$ Academy of Medical Sciences, Zhengzhou University, Zhengzhou, Henan 450000, P.R. China
}

Received January 24, 2018; Accepted June 13, 2018

DOI: $10.3892 /$ or.2018.6533

\begin{abstract}
Since cyclin-dependent kinases 4/6 (CDK4/6) play pivotal roles in cell cycle regulation and are overexpressed in human skin cancers, CDK4/6 inhibitors are potentially effective drugs for skin cancer. In the present study, we present a mixed computational and experimental study attempting to repurpose approved small-molecule drugs as dual CDK4/6 inhibitors for skin cancer treatment. We performed structure-based virtual screening using the docking software idock, targeting an ensemble of CDK4/6 structures. We identified and selected nine compounds with significant predicted scores, and evaluated their cytotoxic effects in vitro in A375 and A431 human skin cancer cell lines. Rafoxanide was found to exhibit the highest cytotoxic effects $\left(\mathrm{IC}_{50}: 1.09 \mu \mathrm{M}\right.$ for $\mathrm{A} 375$ and $1.31 \mu \mathrm{M}$ for A431 cells). Consistent with the expected properties of CDK4/6 inhibitors, rafoxanide significantly increased the G1 phase population. Notably, we revealed that rafoxanide specifically decreased the expression of CDK4/6, cyclin D, retinoblastoma protein $(\mathrm{Rb})$ and the phosphorylation of CDK4/6 and $\mathrm{Rb}$. Furthermore, the anticancer effect of rafoxanide was demonstrated in vivo in BALB/C nude mice subcutaneously xenografted with human skin cancer A375 cells. Rafoxanide (40 mg/kg, i.p.) exhibited significant antitumor activity, comparable to that of oxaliplatin $(5 \mathrm{mg} / \mathrm{kg}$, i.p.). The combined
\end{abstract}

Correspondence to: Professor Marie Chia-Mi Lin, Academy of Medical Sciences, Zhengzhou University, 100 Kexue Street, Zhengzhou, Henan 450001, P.R. China

E-mail: mcmlin@163.com

Key words: CDK4/6 inhibitor, rafoxanide, skin cancers administration of rafoxanide and oxaliplatin produced a synergistic therapeutic effect. To the best of our knowledge, the present study is the first to indicate that rafoxanide inhibits CDK4/6 activity and is a potential candidate drug for the treatment of human skin cancer.

\section{Introduction}

More than 100 types of tumors are clinically apparent on the skin. Non-melanoma skin cancer (NMSC) is the most common malignancy in the world, with cutaneous squamous cell carcinomas (cSCCs) constituting $20 \%$ of all NMSC. While cSCCs typically behave in an indolent fashion and can be cured with local destructive or surgical methods $(1,2)$, if the tumor occurs on the face, surgery could potentially reduce the quality of life of the patient. At present, ifosfamide and cisplatin are commonly used chemotherapy drugs for skin cancers. They kill the skin cancer cells by suppressing the synthesis of DNA. However, non-invasive, effective, and targeted skin cancer chemotherapy drugs are lacking.

An essential characteristic of tumors is increased cell proliferation, which is controlled by cell cycle progression. Cyclin-dependent kinases (CDKs) bind to cell cycle proteins (cyclin) to form a complex, and regulate transcription, metabolism, neuro-physiological processes such as differentiation and development (3-12). Disorders of CDK activity directly or indirectly cause cell proliferation, genomic instability (increase DNA mutations and chromosome deletions) and chromosome instability (chromosome number changes), which are critical for the development and progression of cancer $(13,14)$. Among them, CDK4/6 activity is necessary for the regulation of the cell cycle in the G1 phase (15). CDK4/6 have been selected as important cancer therapeutic target proteins, as they are hallmarks of cancers. 
It has been well documented that the expression levels of CDK4/6 are significantly higher in many tumors (16-18). In skin cancers, it has been reported that CDK4 and CDK6 levels are overexpressed in $>90 \%$ of cases (19). Therefore, CDK4/6 are important targets for skin cancer drugs. Recently, two CDK4/6 inhibitors have been approved for the treatment of breast cancer. However, there is no study on CDK4/6 inhibitors which focuses on skin cancer.

Using structure-based virtual screening via protein-ligand docking to select candidates from FDA-approved smallmolecule drugs, we previously identified two CDK2 inhibitors, adapalene (20) and fluspirilene (21). In the present study, we attempted to identify drugs with the ability to specifically inhibit both CDK4/6 at the same time without affecting CDK2. To achieve this purpose, the free and open-source docking software idock v2.2.1 $(22,23)$ developed by our group, was applied to dock small molecule compounds onto each and all of the available CDK4/6 structures, and to predict their binding conformations, as well as their binding affinities. The top compounds displaying the highest average predicted binding free energy were screened for their ability to reduce the viability of human skin cancer cells. Among them, rafoxanide was discovered as a CDK4/6 dual-inhibitor with the highest anticancer effects in A375 and A431 human skin cancer cell lines. The anticancer effects were also validated in vivo in BALB/C nude mice subcutaneously xenografted with A375 cells.

At present, rafoxanide is mainly used for fasciola hepatica infection $(24,25)$. The present study is the first to indicate that rafoxanide inhibits CDK4/6 and is a potential candidate drug for the treatment of human skin cancer.

\section{Materials and methods}

Ethics statement. The present study, was approved by the Ethics Committee of Yunnan University of Chinese Traditional Medicine (Kunming, China).

Docking. We first collected five X-ray crystallographic structures of CDK4 and $8 \mathrm{X}$-ray crystallographic structures of CDK6 in a complex with a ligand from the Protein Data Bank (PDB) (26-27), and then the co-crystallized water and ligand molecules were deleted manually. Subsequently, the structures of 3,167 approved drugs were gathered from the ZINC database $(28,29)$. To predict the binding conformations and the binding affinities of these drugs with the CDK4/6 proteins, the free and popular docking software idock v2.2.1 $(20,21)$ was used to dock all of the compounds onto all of the CDK2/4/6 structures. Prioritized in accordance with the average predicted binding affinity, 9 commercially available compounds were identified, purchased and subsequently evaluated.

Chemicals and antibodies. We purchased lifibrate, nizofenone, pimozide, trifluperidol, tosufloxacin, cloricromene, sulconazole, sertindole, rafoxanide, oxaliplatin from Sigma-Aldrich (Merck KGaA, Darmstadt, Germany). The antibodies used in the present study, anti-cyclin D (cat. no. ab134175), anti-CDK2/4/6 (cat. nos. ab32147, ab108357 and ab124821), anti-Rb (cat. no. ab181616), anti-phosphorylated (pho)-CDK2/6 (cat. nos. ab76146 and ab194871), anti-pho-Rb (cat. no. ab184796) and GAPDH (cat. no. ab8245) were obtained from Abcam (Cambridge, MA, USA); pho-CDK4 (cat. no. AP0593) was obtained from ABclonal (Wuhan, China).

Cell lines and cell culture conditions. The skin cancer A431 and A375 cell lines were obtained from the Library of the Chinese Academy of Sciences Committee on Type Culture Collection of Cells (Shanghai, China). These cells were cultured in Dulbecco's modified Eagle's medium (DMEM; GE Healthcare Life Sciences, Shanghai, China) containing $10 \%$ fetal bovine serum (FBS; Gibco; Thermo Fisher Scientific, Inc., Waltham, $\mathrm{MA}, \mathrm{USA})$ at $37^{\circ} \mathrm{C}$, in $5 \% \mathrm{CO}_{2}$ and $95 \%$ humidified air.

Cell culture experimental conditions. Briefly, cells were plated in 96-, 24- or 6-well plates (Wuxi Nest Biotechnology Co., Ltd., Wuxi, China) with medium for $24 \mathrm{~h}$. Subsequently, they were treated with medium containing $8 \% \mathrm{FBS}$ and the test compounds at various concentrations $(3,10$ and $30 \mu \mathrm{M} / \mathrm{l})$ and incubation times $(24,48$ and $72 \mathrm{~h})$ as indicated.

MTS assay. Cell proliferative ability was analyzed at various time-points (days 1, 3 and 6) using colorimetric [3-(4,5-dimethylthiazol-2-yl)-5-(3-carboxy methoxy phenyl)2-(4-sulfophenyl)-2H-tetra zolium] (MTS) assay (Promega Corp., Beijing, China). Cells were seeded at an initial density of $9 \times 10^{3}$ cells/well in 96-well plates, and cultured under $5 \% \mathrm{CO}_{2}$ at $37^{\circ} \mathrm{C}$. After a $24-\mathrm{h}$ incubation, the media was replaced with fresh growth media containing testing compounds at various concentrations $(3,10$ and $30 \mu \mathrm{M} / \mathrm{l})$ and incubation times (24, 48 and $72 \mathrm{~h}$ ) as indicated. At the end of the reactions, $20 \mu \mathrm{l}$ of a pre-diluted MTS solution was introduced to each well and the cells were incubated at $37^{\circ} \mathrm{C}$ in the dark for $2 \mathrm{~h}$. The absorbance was recorded at $490 \mathrm{~nm}$ with a Synergy 2 microplate reader (Multiskan FC; Thermo Fisher Scientific, Inc.).

Cell cycle analysis. We conducted cell cycle analysis using an EPICS XL4 Flow Cytometer (Beckman Coulter, Inc., Brea, CA, USA). The cell cycle phase distribution was analyzed using ModFit LT 2.0 software (Verity Software House, Topsham, ME, USA). Briefly, cells $\left(4 \times 10^{4}\right)$ were seeded in 24 -well plates in DMEM containing $0.125 \%$ FBS. After $24 \mathrm{~h}$, the cell culture medium was replaced with DMEM containing $10 \%$ FBS and various doses of rafoxanide $(1,3,10$ or $30 \mu \mathrm{M})$ for 6,12 or $24 \mathrm{~h}$, as indicated. At the end of the experiments, cells $\left(1 \times 10^{4}\right)$ were fixed in ice-cold $70 \%$ ethanol, and stained using a Coulter DNA Prep Reagent kit (Beckman Coulter), and cellular DNA content was assessed. All data were obtained from two separate experiments performed in triplicate.

Cell apoptosis analysis. Apoptosis was determined by staining cells with both Annexin $\mathrm{V}$ and propidium iodide (PI) (Annexin V-FITC/PI Kit; 4A Biotech Co., Ltd., Beijing, China), according to the manufacturer's instructions to quantify the apoptotic cells. Briefly, cells were plated on 24-well plates with DMEM containing $0.125 \%$ FBS. After $24 \mathrm{~h}$, the medium was replaced with DMEM with $10 \% \mathrm{FBS}$, and 3,10 or $30 \mu \mathrm{M}$ of rafoxanide for various time-points $(6,12$ and $24 \mathrm{~h})$ as indicated. At the end of the experiments, cells were trypsinized with $0.25 \%$ trypsin in the absence of ethylenediaminetetraacetic 
Table I. The nine top-scoring compounds purchased and tested in vitro.

\begin{tabular}{lrcclc}
\hline Compound name & ZINC ID & Average score $(\mathrm{kcal} / \mathrm{mol})$ & MW (g/Mol) & \multicolumn{1}{c}{ Clinic usage } & (Refs.) \\
\hline Lifibrate & 537906 & -8.5 & 410.295 & Hypolipidemic agent & $(30)$ \\
Nizofenone & 538096 & -7.14 & 412.87 & Ameliorated ischemic brain damage & $(31)$ \\
Pimozide & 19796084 & -9.99 & 461.55 & Neuroleptic drug & $(32)$ \\
Trifluperidol & 538505 & -7.67 & 409.417 & Schizophrenia drug & $(33)$ \\
Tosufloxacin & 21983589 & -9.56 & 404.34 & Antibiotics & $(34)$ \\
Cloricromene & 576842 & -7.06 & 395.88 & Coronary dilating agent & $(35)$ \\
Sulconazole & 601250 & -8.13 & 460.76 & Antifungal agents & $(36)$ \\
Sertindole & 538337 & -9.48 & 440.94 & Antipsychotic drugs & $(37)$ \\
Rafoxanide & 4181896 & -8.68 & 626.01 & Treatment of Fasciola hepatica infection & $(28)$ \\
\hline
\end{tabular}

The idock score is an estimation of binding free energy in $\mathrm{kcal} / \mathrm{mol}$ units. A more negative value implies a higher predicted binding affinity.

acid (EDTA), washed with PBS twice, and suspended in $500 \mu 1$ of binding buffer. Two microliters of Annexin V-EGFP and $5 \mu \mathrm{l}$ of PI were added to the suspension $\left(2 \times 10^{5}-10^{6}\right.$ cells $\left./ \mathrm{ml}\right)$ and incubated for 5 to $15 \mathrm{~min}$ in the dark. The apoptotic cells were analyzed by flow cytometry (CyFlow Space; Sysmex Partec, Hamburg, Germany).

Western blot analysis. A431 and A375 cells were plated on 6-well plates in DMEM containing $0.125 \%$ FBS. After $24 \mathrm{~h}$, the cell culture media were replaced with DMEM containing $10 \%$ FBS medium containing rafoxanide at concentrations of 3,10 and $30 \mu \mathrm{M}$. Cells were harvested after $6 \mathrm{~h}$ of incubation. As positive controls, CDK2/4/6siRNAs were used to inhibit proteins CDK2,4,6 in A431 and A375 cells. The siRNA oligo sequences were as follows: siRNA-CDK2, 5'-AGTTGT ACCTCCCCTGGAT-3'; siRNA-CDK4, 5'-CAGAUCUCG GUGAACGAUGdTdT-3'; siRNA-CDK6, 5'-GAUGUUGAU CAACUAGGAATT-3'. Briefly, when cells were treated with 40 pmol siRNA of CDK2/4/6/ they were delivered using $5 \mu 1$ RNAiMAX transfection reagent for transfection in each well of the 6-well plates. Cells were lysed in radioimmunoprecipitation assay buffer (Beyotime Institute of Biotechnology, Jiangsu, China), and protein concentrations were assessed using a Bicinchoninic Acid Protein Assay kit (Thermo Fisher Scientific, Inc.). Equal amounts of protein (30 $\mu \mathrm{g} / \mathrm{lane})$ samples were resolved by $10 \%$ SDS-PAGE and transferred onto membranes (Immobilon FL; Merck Millipore). After blocking, the membranes were incubated first with the primary antibodies (Abcam) at $4^{\circ} \mathrm{C}$ overnight, washed in TBS, and then the secondary antibodies (1:4,000; cat. no. SE12; Beijing Solarbio Science and Technology Co., Ltd.,Beijing, China) were added for $1 \mathrm{~h}$ at room temperature. Prior to drying in the dark, the membranes were then washed in TBS+0.1\% Tween, and then $\mathrm{dH}_{2} \mathrm{O}$. The blots were detected using a chemiluminescence detection system (Amersham Biosciences, Piscataway, NJ, USA) (VisionWorks acquisition and analysis, Compatible with Microsoft Windows 7, Vista, XP SP2 or later).

Evaluation of the therapeutic effect of Rafoxanide in vivo in nude mice xenografted with A375 cells. In the present study, we used female BALB/C nude mice $(\mathrm{n}=50$; weighing $15 \mathrm{~g}$; 4-5 weeks old; Vital River Laboratory Animal Technology
Co., Ltd., Beijing, China). The mice were housed under specific pathogen-free conditions in an environment with a 12-h light/dark cycle and 50-80\% humidity. Mice were cared for in accordance with the guidelines of the Laboratory Animal Ethics Committee of Kunming Medical University (Kunming, China). The present study, was approved by the Ethics Committee of Yunnan University of Chinese Traditional Medicine (Kunming, China). A375 cells (1x106) were suspended in $0.2 \mathrm{ml} \mathrm{PBS}$, and injected subcutaneously into the right flank of the mice $(n=3)$. Tumor size was assessed using a caliper. When the tumors grew to $80-100 \mathrm{~mm}^{3}$ (1 week after inoculation), mice were divided randomly into different experimental groups ( $5 \mathrm{mice} / \mathrm{group}$ ), and intraperitoneally injected daily for 21 days with various testing compounds. The mice were sacrificed by cervical dislocation, and the tumors were excised, weighed, and images were captured by camera (Canon, Inc., Tokyo, Japan). The tumor volume was calculated using the formula $\mathrm{V}=\mathrm{ab}^{2} / 2$ ( $\mathrm{a}=$ longest $\mathrm{axis} ; \mathrm{b}=$ shortest axis).

Statistical analysis. Data were obtained from at least three experiments, and values were expressed as the mean \pm standard deviation (SD). Statistical analysis was performed by ANOVA followed with Fisher's least significant difference post hoc tests (version 16.0, SPSS software). $\mathrm{P}<0.05$ was considered to indicate a statistically significant difference between values.

\section{Results}

Identification of candidate CDK4/6 inhibitors using structure-based virtual screening. A total of 3,167 drugs approved by worldwide authorities constituted a library of compounds for screening. They were individually docked to the ATP binding pockets of CDK4/6, and then sorted in the ascending order of their predicted binding free energy. The high-scoring compounds were manually examined based on in silico estimations of binding strength, appropriate molecular weight and other drug-like properties, and complementary matching of molecular shape. Finally, high-scoring compounds were selected and 9 commercially available compounds [Table I (28,30-37)] were purchased for subsequent wet-lab validations. 

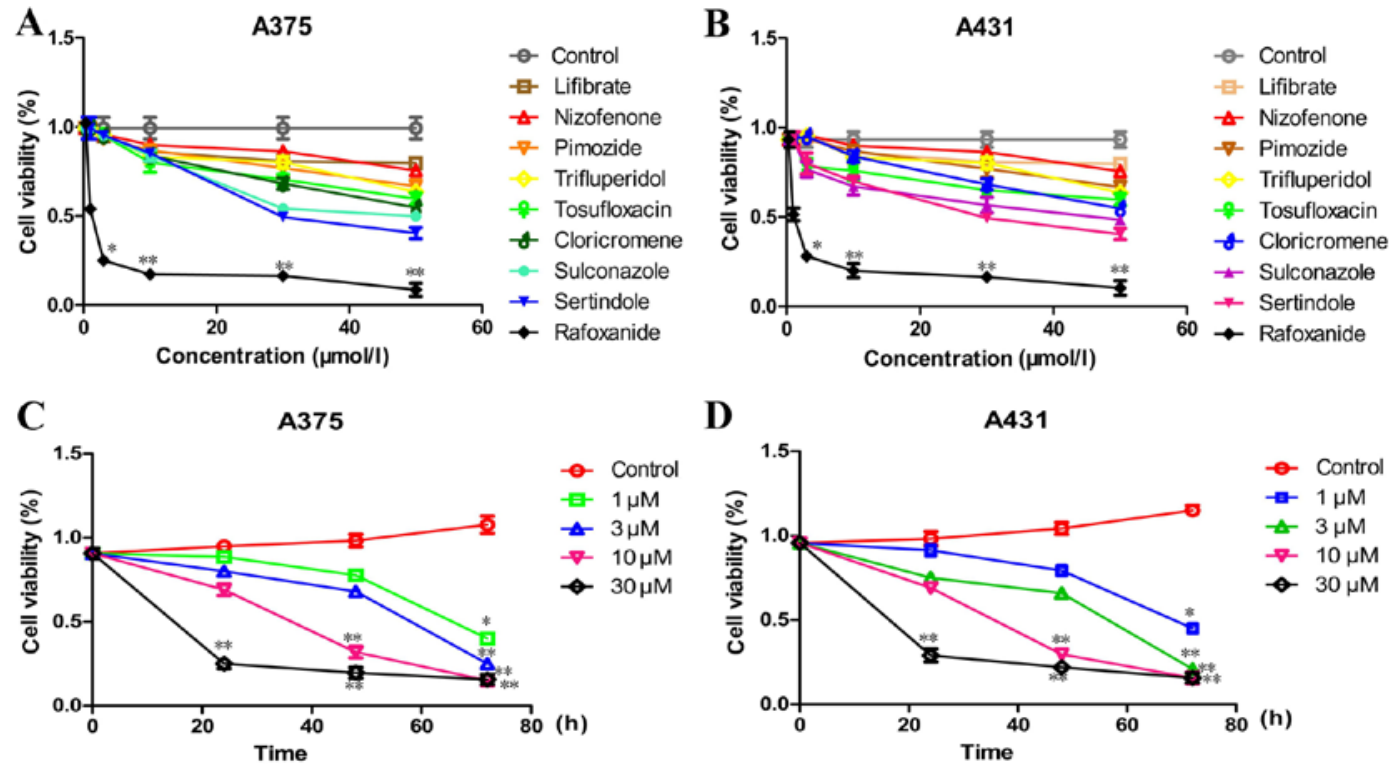

Figure 1. Comparison of the effect of nine candidate CDK4/6 inhibitors on the viability of A375 and A431 skin cancer cells. (A and B) The cell viability assay. Nine compounds had discrepant cytotoxicity to the A375 and A431 cell lines at different concentrations as determined by an MTT assay, with rafoxanide exhibiting the greatest cytotoxicity compared with the control $\left({ }^{*} \mathrm{P}<0.05,{ }^{* *} \mathrm{P}<0.01\right)$. (C and $\left.\mathrm{D}\right)$ Rafoxanide exhibited dose- and time-dependent inhibition on cell viability in A375 and A431 cell lines compared with the control ( $\left.\mathrm{P}<0.05,{ }^{* *} \mathrm{P}<0.01\right)$.
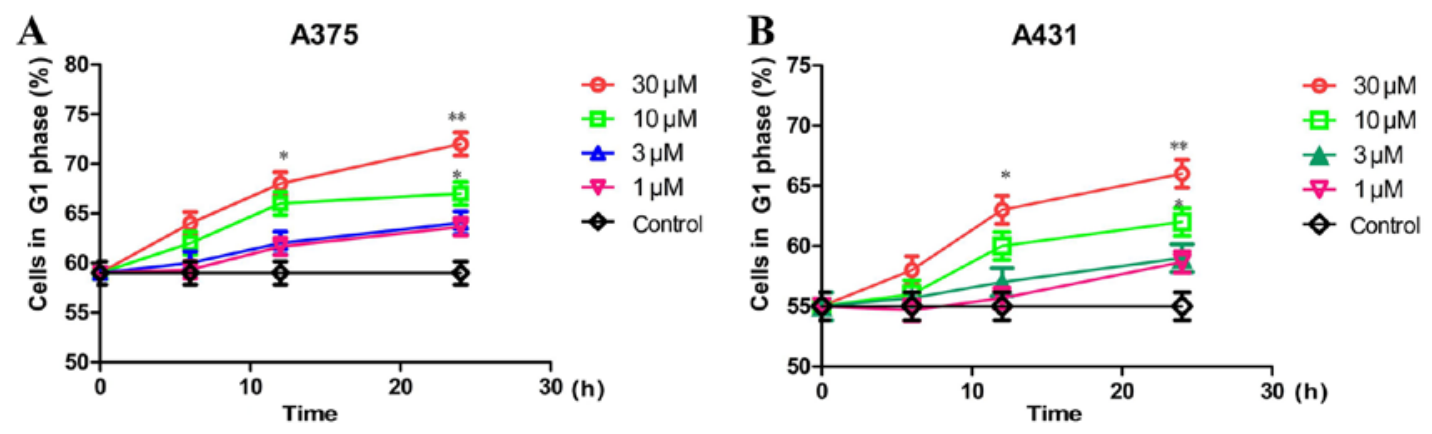

Figure 2. Effects of rafoxanide on the cell cycle distribution in A375 and A431 skin cancer cells. (A) A375 and (B) A431 cell lines were treated with different concentrations $(1,3,10$ and $30 \mu \mathrm{M})$ of rafoxanide for 6,12 and $24 \mathrm{~h}$, and the cell cycle distributions were determined by flow cytometry. Rafoxanide treatment dose- and time-dependently increased the percentage of cells in the G1 phase. Concentrations at 10 and $30 \mu \mathrm{M}$ significantly increased the percentage of cells in the G1 phase at $24 \mathrm{~h}$, compared to the control $\left({ }^{*} \mathrm{P}<0.05,{ }^{* *} \mathrm{P}<0.01\right)$.
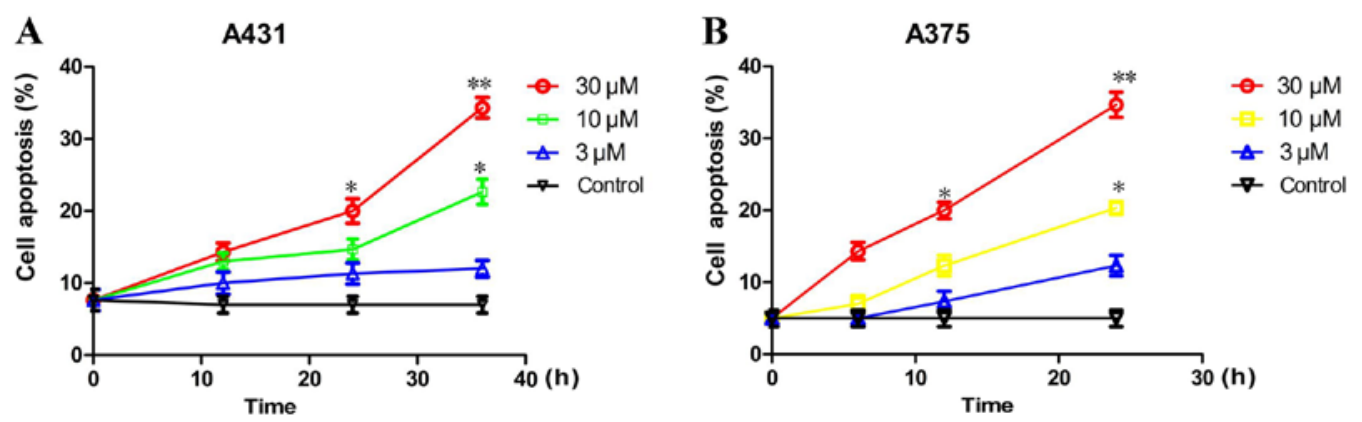

Figure 3. Rafoxanide treatment induces cell apoptosis. Rafoxanide treatment at concentrattions of 30 or $10 \mu \mathrm{M}$ significantly increased the percentage of apoptosis in (A) A431 and (B) A375 cell lines compared to the control in a dose- and time-dependent manner ( $\left.{ }^{*} \mathrm{P}<0.05,{ }^{* *} \mathrm{P}<0.01\right)$.

Rafoxanide treatment markedly decreases the viability of A375 and A431 human skin cancer cells. We first evaluated the cytotoxic effects of the nine compounds using MTS assays in two skin cancer cell lines. All nine compounds (lifibrate, nizofenone, pimozide, trifluperidol, tosufloxacin, cloricromene, sulconazole, sertindole and rafoxanide) decreased the viability of the $\mathrm{A} 375$ and $\mathrm{A} 431$ cells. The $\mathrm{IC}_{50}$ values were calculated using GraphPad Prism 5 (GraphPad Software, Inc., La Jolla, CA, USA). Among them, rafoxanide exhibited the lowest $\mathrm{IC}_{50}$ value $(1.09 \mu \mathrm{M}$ for A375 and $1.31 \mu \mathrm{M}$ 
A
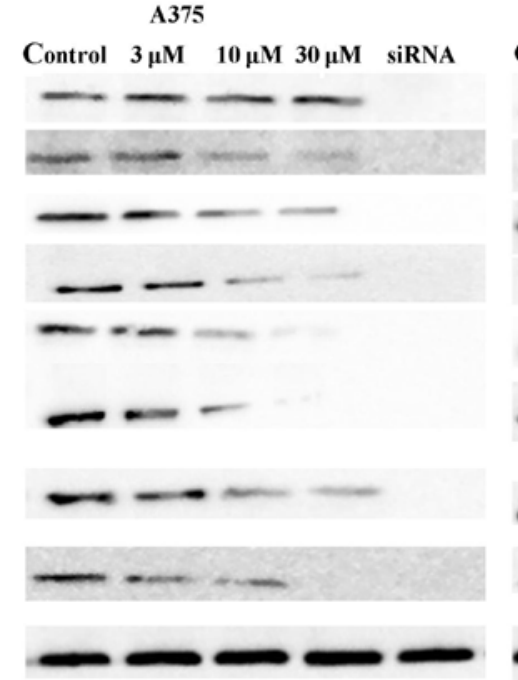

B Protein level of CDK2

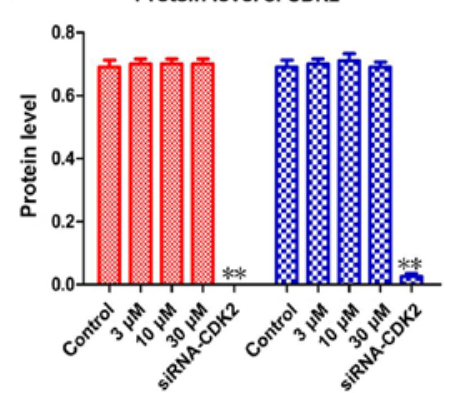

D
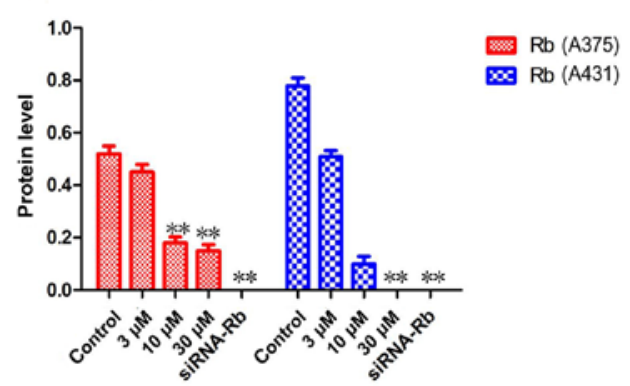

A431

Control $3 \mu \mathrm{M} \quad 10 \mu \mathrm{M} 30 \mu \mathrm{M} \quad$ siRNA

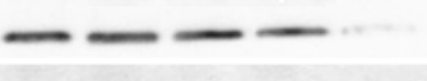

CDK2

Cyclin D

Pho-CDK6

CDK6

Pho-CDK4

CDK4

Rb

Pho-Rb

GAPDH

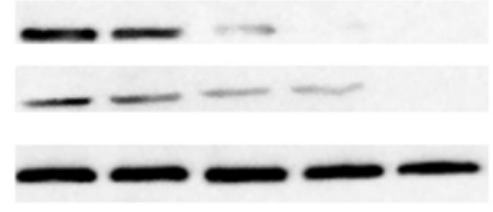

C Protein level of CDK6

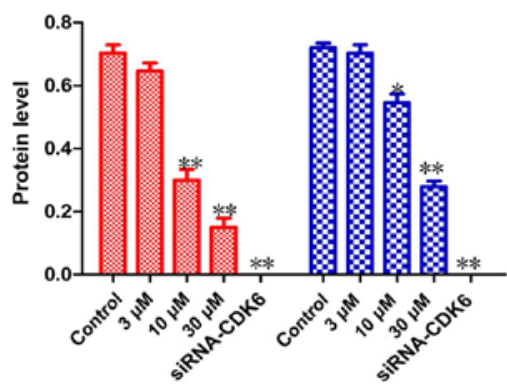

E Protein level of CDK4

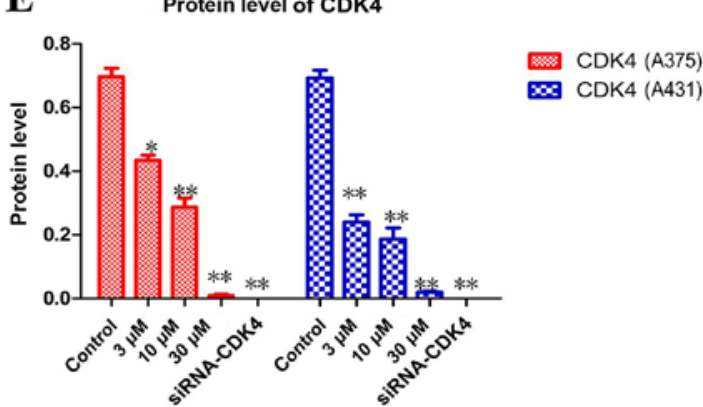

Figure 4. Effects of rafoxanide treatment on the expression of cyclin D, CDK4/6 and Rb. (A) A375 and A431 cells were plated on 6-well plates with 0.125\% FBS medium for $24 \mathrm{~h}$ and then with $10 \%$ FBS medium containing rafoxanide at concentrations of 3,10,30 $\mu \mathrm{M}$. Cells were harvested after a 6-h incubation and proteins were analyzed by western blotting. Western blotting results revealed that rafoxanide treatment significantly reduced the expression of CDK4/6, pho-CDK4/6, Rb, pho-Rb, cyclin D in A375 and A431 compared to the control. (B) Graph indicated the expression protein level of CDK2 was not affected by treatment with rafoxanide. (C-E) Graphs revealing the dose-dependent reduction on the protein expression level of CDK4, CDK6 and Rb in the A375 and A431 cell lines compared to the control ( $\left.{ }^{*} \mathrm{P}<0.05,{ }^{* *} \mathrm{P}<0.01\right)$. Based on these results, we proposed that rafoxanide reduced the activity of CDK4/6, which resulted in decreased binding and increased degradation of cyclin D. Furthermore, it suppressed Rb and Rb phosphorylation, and reduced the activation of E2F1. These functions caused cell cycle G1-arrest and reduced cell cycle progression from the G1 to the S phase.

for A431 cells, respectively). As displayed in Fig. 1 rafoxanide exhibited the strongest cytotoxic effects in A375 (Fig. 1A) and A431 (Fig. 1B) cells compared to the other 8 compounds. The growth inhibitory effect of rafoxanide was dose- and time-dependent, with significant inhibition observed at concentrations of $\geq 3 \mu \mathrm{M}$ in A375 cells (Fig. 1C) and A431 cells (Fig. 1D).

Rafoxanide treatment causes cell cycle arrest in the G1 phase. We then assessed the effect of rafoxanide treatment in the inhibition of cell cycle progression. The A375 and A431 cells were treated with various doses of rafoxanide $(3,10$ or $30 \mu \mathrm{M})$ for different time-points $(6,12$ or $24 \mathrm{~h})$, and their effects on cell cycle profiles were assessed using flow cytometric analysis. After $24 \mathrm{~h}$ of incubation, our results indicated that rafoxanide treatment dose- and time-dependently $(\mathrm{P}<0.05)$, significantly increased the G1 phase populations, which was accompanied by concurrent decreased $\mathrm{S}$ - and $\mathrm{G}_{2} / \mathrm{M}$ phase populations, in A375 (Fig. 2A) and A431 (Fig. 2B) cells.

Rafoxanide treatment stimulates cell apoptosis. In addition, we investigated whether rafoxanide could stimulate cell apoptosis using flow cytometry with an Annexin V-FITC/PI kit (4A Biotech Co., Ltd., Beijing, China). As displayed in Fig. 3, 


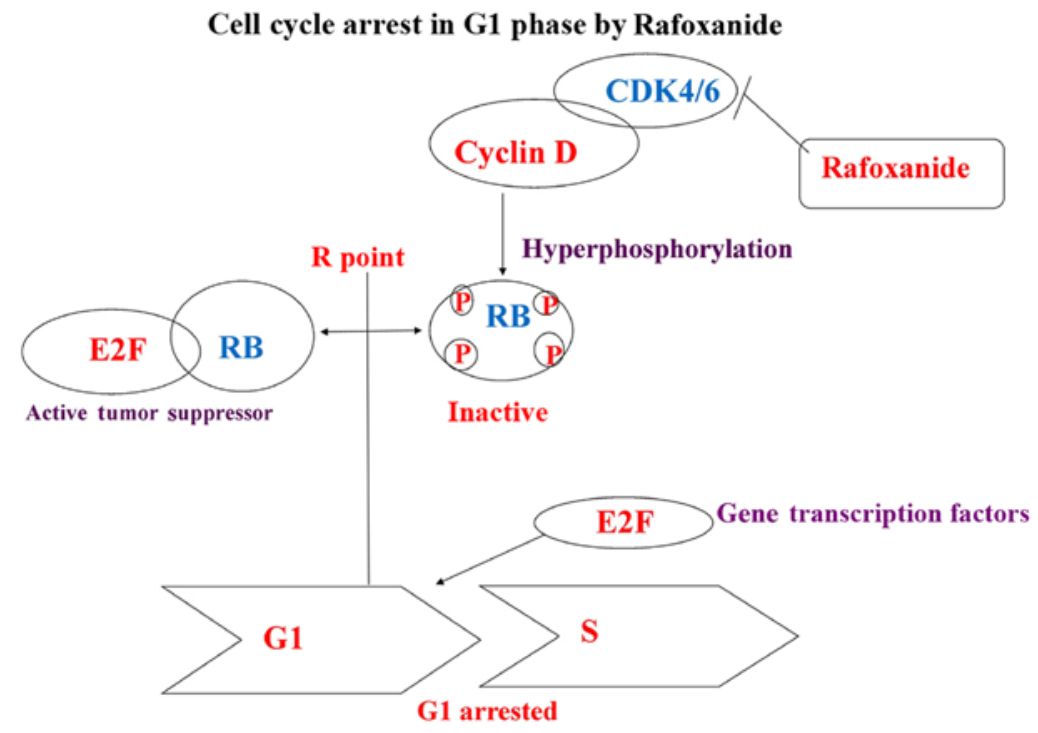

Figure 5. Mechanisms of rafoxanide. Cell cycle regulation via cyclin-CDK complexes. Phosphorylation of pRB via CDK4/6 releases pRB from its association with transcription factor E2F, allowing the cell cycle to proceed. CDK4/6 inhibition by rafoxanide prevents phosphorylation of pRB, resulting in cell cycle arrest.

rafoxanide treatment at 10 and $30 \mu \mathrm{M}$ concentrations significantly increased the percentage of apoptosis in A375 (Fig. 3A) and A431 cells (Fig. 3B) in a dose- and time-dependent manner $(\mathrm{P}<0.05)$.

Rafoxanide decreases the expression of $C D K 4 / 6, R b$, cyclin D, pho-CDK4/6 and pho-Rb, but not CDK2 in A375 and A431 cells. To demonstrate the mechanism of actions, we investigated the effects of rafoxanide on the expression of key proteins involved in cell cycle G1 phase progression, including CDK2/4/6, cyclin $\mathrm{D}$, Rb, pho-CDK2/4/6 and pho- $\mathrm{Rb}$ by western blot analysis in A375 and A431 cells (Fig. 4). The results revealed that rafoxanide specifically reduced the expression of CDK4/6, cyclin D, Rb, pho-CDK4/6, pho-Rb, in the A375 and A431 cells. Notably, the expression level of CDK2 was not affected. These patterns are consistent with what is expected for a specific CDK4/6 dual inhibitor. Based on these results, we proposed that rafoxanide reduced the activity of CDK4/6, which decreased the binding of cyclin D and promoted degradation, suppressed $\mathrm{Rb}$ and $\mathrm{Rb}$ phosphorylation, and inhibited the activation of E2F1-caused cell cycle $\mathrm{G}_{1}$ arrest and suppressed cell cycle progression from the $\mathrm{G}_{1}$ to $\mathrm{S}$ phases (7-12) (Fig. 5).

Rafoxanide (i.p.) administration reduces the growth of tumors subcutaneously xenografted with A375 cells in vivo in $B A L B / C$ nude mice. To assess the in vivo effect of rafoxanide, a mouse model of A375 cell xenografted skin tumors was established in BALB/C nude mice. Following the appearance of the tumors, tumor volumes were assessed every 3-4 days. Seven days after tumor inoculation, the tumor volume reached $80-100 \mathrm{~mm}^{3}$, and the animals were divided into 4 groups ( 5 mice/group) and were administered daily for 21 days by i.p. injection the following: i) PBS (control group); ii) oxaplatin $(5 \mathrm{mg} / \mathrm{kg})$; iii) rafoxanide $(40 \mathrm{mg} / \mathrm{kg})$; and iv) combination of rafoxanide $(40 \mathrm{mg} / \mathrm{kg})$ and oxaplatin $(5 \mathrm{mg} / \mathrm{kg})$. As displayed in Fig. 6A, our results demonstrated that the
A

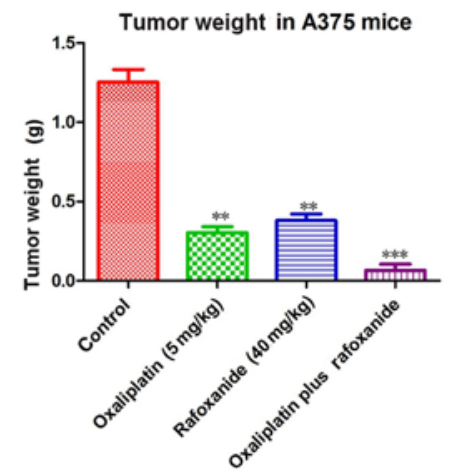

B

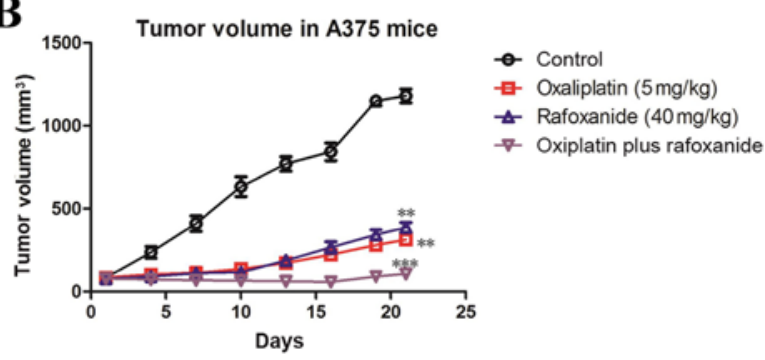

C

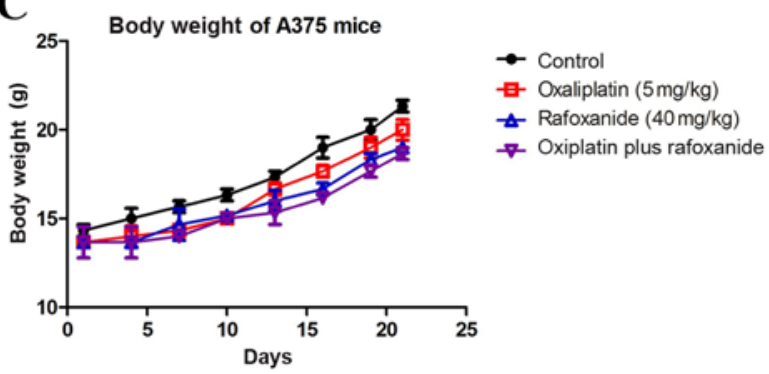

Figure 6. Rafoxanide (i.p.) combined with oxaliplatin (i.p.) treatment significantly reduces tumor growth in vivo in nude mice xenografted with A375 cells. (A) Significant reduction of tumor weight was observed upon the administration of rafoxanide (i.p.) $40 \mathrm{mg} / \mathrm{kg}$ combined with oxaliplatin (i.p.) $5 \mathrm{mg} / \mathrm{kg}$ compared with the control on day $\left.21{ }^{* * *} \mathrm{P}<0.01,{ }^{* * *} \mathrm{P}<0.01\right)$. (B) The volume of the tumors significantly decreased upon the administration of rafoxanide (i.p.) $40 \mathrm{mg} / \mathrm{kg}$ combined with oxaliplatin (i.p.) $5 \mathrm{mg} / \mathrm{kg}$ treatment compared with rafoxanide (i.p.) $40 \mathrm{mg} / \mathrm{kg}$ only or oxaliplatin (i.p.) $5 \mathrm{mg} / \mathrm{kg}$ only on day $21\left({ }^{* *} \mathrm{P}<0.01,{ }^{* * *} \mathrm{P}<0.01\right)$. (C) No significant body weight changes were observed in all four groups $(\mathrm{P}>0.05)$. 
potency of rafoxanide $(40 \mathrm{mg} / \mathrm{kg})$ was similar to that of oxaplatin $(5 \mathrm{mg} / \mathrm{kg})$ in reducing tumor weight. The combined administration of both drugs produced the greatest therapeutic effect. In addition, the effect of rafoxanide $(40 \mathrm{mg} / \mathrm{kg})$ was similar to that of oxaplatin $(5 \mathrm{mg} / \mathrm{kg})$ in reducing tumor volume, with combined administration producing the greatest therapeutic effect (Fig. 6B). We also assessed the body weight of all the mice in the four groups. No significant body weight changes were observed in all four groups $(\mathrm{P}>0.05)$ (Fig. 6C). In addition, we did not detect any obvious toxicity in mice during the course of the experiments.

\section{Discussion}

The most common forms of skin cancer include basal cell carcinoma, squamous cell carcinoma and melanoma. Early skin cancer can be cured by surgical resection, and $\sim 80 \%$ of cases are treated using this method. However, in cases where skin cancer is on the face, surgical resection would decrease the quality of life of the patient. Thus, new drugs are urgently needed (38).

Aberrant cell cycle progression and uncontrolled cell proliferation is an indication of cancer (39). CDK4/6 can drive cell cycle progression from the G1 to the S phase, thus, dysregulation of CDKs plays a central role in tumorigenesis. CDK4/6 was revealed to be overexpressed in more than $90 \%$ of skin cancers $(40,41)$. The CDK4/6 activating events in skin cancer render it a potential therapeutic target, therefore a dual-inhibitor targeting the activities of CDK4 and CDK6 could be an effective agent to reduce tumor cell proliferation in human neoplasms.

In the present study, we performed virtual screening to search for a specific CDK4/6 dual inhibitor from a library of FDA-approved small molecule drugs. We reveal in the present study for the first time, that rafoxanide exhibited dual CDK4/6 inhibitor activity in vitro in human skin cancer cells. Furthermore, rafoxanide $(40 \mathrm{mg} / \mathrm{kg}$, i.p.) exhibited significant in vivo anticancer efficacy, which was comparable to that of the clinically used anticancer drug oxaliplatin $(5 \mathrm{mg} / \mathrm{kg})$ in nude mice subcutaneously xenografted with A375 skin cancer cells. The combination of rafoxanide and oxaliplatin produced the greatest therapeutic effect.

Three CDK4/6 inhibitors: Palbociclib, ribociclib and abemaciclib, have been tested in randomised phase II/III trials of clinical breast cancer (BC). Tests results revealed significantly increased progression-free survival in first- and second-line treatment for advanced hormone receptor-positive HER2-negative BC (42). The Food and Drug Administration (FDA) and the European Commission (EMA) have approved palbociclib for the treatment of patients $\mathrm{HR}^{+} \mathrm{HER} 2^{-}$in locally advanced or metastatic $\mathrm{BC}(\mathrm{aBC})$, in combination with an aromatase inhibitor as initial therapy in postmenopausal women or in combination with fulvestrant in women who have received prior endocrine therapy. Ribociclib has been approved by the FDA in combination with an aromatase inhibitor as an initial therapy for postmenopausal women with $\mathrm{HR}^{+}$HER2- in BC (43). However, the adverse effects of these agents were neutropenia, infections, fatigue and gastrointestinal toxicity. Furthermore, the effect of CDK4/6 inhibitors was dependent on an intact, functional $\mathrm{Rb}$ group $(42,43)$.
The incidence of $\mathrm{Rb}$ loss was dependent on the clinical subtype and was more common in certain subtypes. It has been reported that $\mathrm{Rb}$ loss was observed at a percentage of $20-30 \%$ in different subtypes of cancer $(44,45)$. Skin cancer has been reported to have $33 \%$ of Rb loss (46). It remains to be determined whether the effect of rafoxanide is dependent on an intact functional $\mathrm{Rb}$ group.

Rafoxanide has been tested and revealed to have no obvious toxic effects by oral administration at $6.7 \mathrm{mg} / \mathrm{kg}$ in rabbits infected with 2-, 4-, 6- or 8-week old Fasciola hepatica (47). The present study did not observe any significant toxicity and change in the body weight of the BALB/C nude mice administered (i.p.) with rafoxanide $(40 \mathrm{mg} / \mathrm{kg})$ over 21 days. These results suggested that i.p. injection of rafoxanide was relatively safe. The toxicity of rafoxanide for skin cancer therapy requires further investigations.

In addition to inhibiting CDK4/6, rafoxanide was reported to be a potent uncoupling factor of mitochondrial oxidative phosphorylation and exert the effects by interfering with parasite mitochondrial ATP synthesis (48). The possible function and effect of rafoxanide as an uncoupling agent of mitochondrial oxidative phosphorylation, and whether it would interfere with mitochondria ATP synthesis in skin cancer cells remain to be determined. In addition, our results revealed that rafoxanide also promoted apoptosis in skin cancer cells. While the mechanisms are not known, it is possible that rafoxanide may induce apoptosis via the E2F-p53-Bax/caspase 3 signaling pathway (49-51). In the next phase of the study, the mechanisms of rafoxanide-induced apoptosis in skin cancer will be identified by microarray and other methods.

At present, rafoxanide is used for the treatment of Fasciola hepatica infection $(28,29)$. As an FDA-approved drug, rafoxanide alone or in combination with other chemotherapeutic drugs may be suitable for the treatment of skin cancers. Further modifications may be required to improve its efficacy and reduce its toxicity, including chemical modification and/or packaging rafoxanide in nanoparticles or liposomes. The possibility that rafoxanide could be considered as either an alternative or a complementary therapy with surgery for the treatment of skin cancer, particularly when occurring on the face, warrants further investigations.

\section{Acknowledgements}

Not applicable.

\section{Funding}

The present study was supported by grants from the Hsiang-fu Kung Academician Workstation of Kunming Medical University (no. NSFC 81272549), the Natural Science Foundation of SZU (no. 827-000100 starting project of Shenzhen high-level overseas talent), the Shenzhen Basic Research Project (JCYJ20160331114230843 and JCYJ20150324141711558), the Guizhou Science and Technology Department [no. QKHJC (2017) 1171], the Chinese Ministry of Science and Technology (no. 2016YFC0904600), the Vice-Chancellor's One-off Discretionary Fund, Faculty of Social Science Postdoctoral Fellowship Scheme and Institute of Future Cities and the Chinese University of Hong Kong 
and the joint application project from the Yunnan Provincial Science and Technology Department (2014FB060).

\section{Availability of data and materials}

The datasets used during the present study are available from the corresponding author upon reasonable request.

\section{Authors' contributions}

MCML, HFK, KSL, MHW conceived and designed the study. XL, LLi, LLe, QH, WC, AS and HY conducted the research. $\mathrm{KK}, \mathrm{CD}, \mathrm{YZ}, \mathrm{YQ}, \mathrm{YD}$ and $\mathrm{YHH}$ performed the analysis of data. $X S$ and HL revised the manuscript and were also involved in the conception of the study. MCML critically revised the article for important intellectual content. All authors read and approved the manuscript and agree to be accountable for all aspects of the research in ensuring that the accuracy or integrity of any part of the work are appropriately investigated and resolved.

\section{Ethics approval and consent to participate}

Mice were cared for in accordance with the guidelines of the Laboratory Animal Ethics Committee of Kunming Medical University (Kunming, China). The present study, was approved by the Ethics Committee of Yunnan University of Chinese Traditional Medicine (Kunming, China).

\section{Patient consent for publication}

Not applicable.

\section{Competing interests}

The authors declare that they have no competing interests.

\section{References}

1. Bikle DD, Jiang Y, Nguyen T, Oda Y and Tu CL: Disruption of vitamin $\mathrm{D}$ and calcium signaling in keratinocytes predisposes to skin cancer. Front Physiol 7: 296, 2016.

2. Fu T, Aasi SZ and Hollmig ST: Management of high-risk squamous cell carcinoma of the skin. Curr Treat Options Oncol 17: $34,2016$.

3. Lim S and Kaldis P: CDKcyclins and CKIs: Roles beyond cell cycle regulation. Development 140: 3079-3093, 2013.

4. Asghar U, Witkiewicz AK,Turner NC and Knudsen ES: The history and future of targeting cyclin Dependent kinases in cancer therapy. Nat Rev Drug Discov 14: 130-146, 2015.

5. Cicenas J, Kalyan K, Sorokinas A, Jatulyte A, Valiunas D, Kaupinis A and Valius M: Highlights of the latest advances in research on CDK inhibitors. Cancers 6: 2224-2242, 2014

6. Canavese M, Santo L and Raje N: Cyclin dependent kinases in cancer: Potential for therapeutic intervention. Cancer Biol Ther 13: 451-457, 2014.

7. Lukas J, Bartkova $\mathbf{J}$ and Bartek J: Convergence of mitogenic signalling cascades from diverse classes of receptors at the cyclin D-cyclin-dependent kinase-pRb-controlled G1 checkpoint. Mol Cell Biol 16: 6917-6925, 1996.

8. Hinz M, Krappmann D, Eichten A, Heder A, Scheidereit C and Strauss M: NF- $\kappa \mathrm{B}$ function in growth control: Regulation of cyclin D1 expression and $\mathrm{G}_{0} / \mathrm{G}_{1}$-to-S-phase transition. Mol Cell Biol 19: 2690-2698, 1999.

9. Weinberg RA: The retinoblastoma protein and cell cycle control. Cell 81: 323-330, 1995.

10. Sherr CJ: Cancer cell cycles. Science 274: 1672-1677, 1996.

11. Malumbres M: Physiological relevance of cell cycle kinases. Physiol Rev 91: 973-1007, 2011.
12. Choi YJ and Anders L: Signaling through cyclin D-dependent kinases. Oncogene 33: 1890-1903, 2014.

13. Pucci B, Kasten M and Giordano A: Cell cycle and apoptosis. Neoplasia 2: 291-299, 2000.

14. Malumbres $\mathrm{M}$ and Barbacid M: Cell cycle, CDKs and cancer: A changing paradigm. Nat Rev Cancer 9: 153-166, 2009.

15. Cohen P: Protein kinase-the major drug targets of the twenty-first century? Nat Rev Drug Discov 1: 309-315, 2002.

16. Li M, Xiao A, Floyd D, Olmez I, Lee J, Godlewski J, Bronisz A, Bhat KPL, Sulman EP, Nakano I and Purow B: CDK4/6 inhibition is more active against the glioblastoma proneural subtype. Oncotarget 8: 55319-55331, 2017.

17. Dall'Acqua A, Sonego M, Pellizzari I, Pellarin I, Canzonieri V, D'Andrea S, Benevol S, Sorio R, Giorda G, Califano D, et al: CDK6 protects epithelial ovarian cancer from platinum-induced death via FOXO3 regulation. EMBO Mol Med 9: 1415-1433, 2017.

18. Malumbres M: Oncogene-induced mitotic stress: p53 and pRb get mad too. Cancer Cell 19: 691-2, 2011.

19. Sheppard KE and McArthur GA: The cell cycle regulator CDK4: An emerging therapeutic target in melanoma. Clin Cancer Res 19: 5320-5328, 2013.

20. Shi XN, Li H, Yao H, Liu X, Li L, Leung KS, Kung HF and Lin MC: Adapalene inhibited the activity of cyclin-dependent kinase 2 in colorectal carcinoma . Mol Med Rep 12: 6501-6508, 2015.

21. Shi XN, Li H, Yao H, Liu X, Li L, Leung KS, Kung HF, Lu D, Wong MH and Lin MC: In silico identification and in vitro and in vivo validation of anti-psychotic drug fluspirilene as a potential CDK2 inhibitor and a candidate anti-cancer drug. PLoS One 10: e0132072, 2015.

22. Li H, Leung KS and Wong MH: Idock: A multithreaded virtual screening tool for flexible ligand docking. In: Proceedings of the 2012 IEEE symposium on computational intelligence in bioinformatics and computational biology (CIBCB), San Diego, pp77-84, 2012.

23. Li H, Leung KS, Ballester PJ and Wong MH: Istar: A web platform for large-scale protein-ligand docking. PLoS One 9: e85678, 2014.

24. Ross DB: Treatment of experimental Fasciola hepatica infection of sheep with rafoxanide. Vet Rec 87: 110-111, 1970.

25. Elitok B, Elitok OM and Kabu M: Field trial on comparative efficacy of four fasciolicides against natural liver fluke infection in cattle. Vet Parasitol 135: 279-285, 2006.

26. Berman HM, Westbrook J, Feng Z, Gilliland G, Bhat TN, Weissig H, Shindyalov IN and Bourne PE: The protein data bank. Nucleic Acids Res 28: 235-242, 2000.

27. Huang $\mathrm{Z}$ and Wong CF: inexpensive method for selecting receptor structures for virtual screening. J Chem Inf Model 56: 21-34, 2016.

28. Irwin JJ and Shoichet BK: ZINC-a free database of commercially available compounds for virtual screening. J Chem Inf Model 45: $177-182,2005$.

29. Irwin JJ, Sterling T, Mysinger MM, Bolstad ES and Coleman RG: ZINC: A free tool to discover chemistry for biology. J ChemInf Model 52: 1757-1768, 2012

30. Arnold A, McAuliff MP and Beyler AL: Metabolic effects of a new hypolipidemic agent, ciprofibrate. J Pharm Sci 68: 1557-1558, 1979.

31. Matsumoto Y, Aihara K, Kamata T and Goto N: Nizofenone, a neuroprotective drug, suppresses glutamate release and lactate accumulation. Eur J Pharmacol 262: 157-161, 1994.

32. Janssen PA, Niemegeers CJ, Schellekens KH, Dresse A, Lenaerts FM, Pinchard A, Schaper WK, van Nueten JM and Verbruggen FJ: Pimozide, a chemically novel, highly potent and orally long-acting neuroleptic drug. I. The comparative pharmacology of pimozide, haloperidol, and chlorpromazine. Arzneimittelforschung 18: 261-279, 1968.

33. Bueno JR: Therapeutic evaluation of R2498 (triperidol) in hospitalized schizophrenic patients. J Bras Psiquiatr 14: 81-91, 1965 (In Italian).

34. Fujimaki K, Noumi T, Saikawa I, Inoue M and Mitsuhashi S: In vitro and in vivo antibacterial activities of T-3262, a new fluoroquinolone. Antimicrob Agents Chemother 32: 827-833, 1988.

35. Dejana E, de Castellarnau C, Balconi G, Rotilio D, Pietra A and de Gaetano G: AD 6, a coronary dilating agent, stimulates PGI2 production in rat aorta ex vivo and in human endothelial cells in culture. Pharmacol Res Commun 14: 719-724, 1982. 
36. Lassus A, Forström S and Salo O: A double-blind comparison of sulconazole nitrate $1 \%$ cream with clotrimazole $1 \%$ cream in the treatment of dermatophytoses. Br J Dermatol 108: 195-198, 1983.

37. Muscatello MR, Bruno A, Micali Bellinghieri P, Pandolfo G and Zoccali RA: Sertindole in schizophrenia: Efficacy and safety issues. Expert Opin Pharmacother 15: 1943-1953, 2014.

38. Gray-Schopfer V, Wellbrock C and Marais R: Melanoma biology and new targeted therapy. Nature 445: 851-857, 2007.

39. Hanahan D and Weinberg RA: Hallmarks of cancer: The next generation. Cell 144: 646-674, 2011.

40. Walker GJ, Flores JF, Glendening JM, Lin AH, Markl ID and Fountain JW: Virtually $100 \%$ of melanoma cell lines harbor alterations at the DNAlevel within CDKN2A, CDKN2B, or one of their downstream targets. Genes Chromosomes Cancer 22: $157-163,1998$.

41. Curtin JA, Fridlyand J, Kageshita T, Patel HN, Busam KJ, Kutzner H, Cho KH, Aiba S, Bröcker EB, LeBoit PE, et al Distinct sets of genetic alterations in melanoma. N Engl J Med 353: 2135-2147, 2005.

42. Polk A, Kolmos IL, Kümler I and Nielsen DL: Specific CDK4/6 inhibition in breast cancer: A systematic review of current clinical evidence. ESMO Open 1: e000093, 2017.

43. de Groot AF, Kuijpers CJ and Kroep JR: CDK4/6 inhibition in early and metastatic breast cancer: A review. Cancer Treat Rev 60: 130-138, 2017.

44. Dean JL, Thangavel C, McClendon AK, Reed CA and Knudsen ES: Therapeutic CDK4/6 inhibition in breast cancer: Key mechanisms of response and failure. Oncogene 29: 4018-4032, 2010.
45. Bosco EE and Knudsen ES: RB in breast cancer: At the crossroads of tumorigenesis and treatment. Cell Cycle 6: 667-671, 2007.

46. Tchernev $\mathrm{G}$ and Orfanos CE: Downregulation of cell cycle modulators p21, p27, p53, Rb and proapoptotic Bcl-2-relatedproteins Bax and Bak in cutaneous melanoma is associated with worse patient prognosis: Preliminary findings. J Cutan Pathol 34: 247-256, 2007.

47. Stammers BM: The effects of rafoxanide and nitroxynil on the survival, growth and morphology of Fasciola hepatica in rabbits. Z Parasitenkd 46: 153-156, 1975.

48. Matsubara K, Sanoh S, Ohta S, Kitamura S, Sugihara K and Fujimoto N: An improved thyroid hormone reporter assay to determine the thyroid hormone-like activity of amiodarone, bithionol, closantel and rafoxanide. Toxicol Lett 208: 30-35, 2012.

49. Xie Y, Si J, Wang YP, Li HY, Di CX, Yan JF, Ye YC, Zhang YS and Zhang $\mathrm{H}$ : E2F is involved in radio resistance of carbon ion induced apoptosis via Bax/caspase 3 signal pathway in human hepatoma cell. J Cell Physiol 233: 1312-1320, 2018.

50. Ma L, Yu HJ, Gan SW, Gong R, Mou KJ, Xue J and Sun SQ: p53-Mediated oligodendrocyte apoptosis initiates demyelination after compressed spinal cord injury by enhancing ER-mitochondria interaction and E2F1 expression. Neurosci Lett 644: 55-61, 2017.

51. Zhan L, Huang C, Meng XM, Song Y, Wu XQ, Miu CG, Zhan XS and Li J: Promising roles of mammalian E2Fs in hepatocellular carcinoma. Cell Signal 26: 1075-1081, 2014. 